\title{
Application of Laboratory and Modeling Capabilities to Extreme Ultraviolet Spectroscopy of Astrophysical Sources
}

\author{
C.W. Mauche, D.A. Liedahl, P. Beiersdorfer
}

February 25, 2000

U.S. Department of Energy

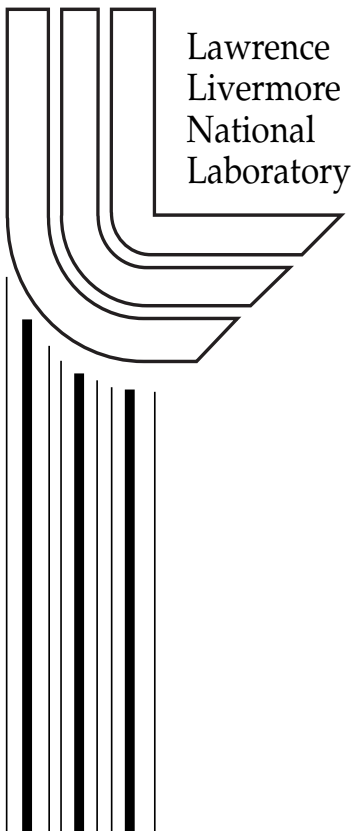




\section{DISCLAIMER}

This document was prepared as an account of work sponsored by an agency of the United States Government. Neither the United States Government nor the University of California nor any of their employees, makes any warranty, express or implied, or assumes any legal liability or responsibility for the accuracy, completeness, or usefulness of any information, apparatus, product, or process disclosed, or represents that its use would not infringe privately owned rights. Reference herein to any specific commercial product, process, or service by trade name, trademark, manufacturer, or otherwise, does not necessarily constitute or imply its endorsement, recommendation, or favoring by the United States Government or the University of California. The views and opinions of authors expressed herein do not necessarily state or reflect those of the United States Government or the University of California, and shall not be used for advertising or product endorsement purposes.

Work performed under the auspices of the U. S. Department of Energy by the University of California Lawrence Livermore National Laboratory under Contract W-7405-Eng-48.

This report has been reproduced directly from the best available copy.

Available to DOE and DOE contractors from the

Office of Scientific and Technical Information

P.O. Box 62, Oak Ridge, TN 37831

Prices available from (423) 576-8401

http://apollo.osti.gov/bridge/

Available to the public from the National Technical Information Service

U.S. Department of Commerce 5285 Port Royal Rd., Springfield, VA 22161 http://www.ntis.gov/

OR Lawrence Livermore National Laboratory Technical Information Department's Digital Library http://www.llnl.gov/tid/Library.html 


\title{
Application of Laboratory and Modeling Capabilities to Extreme Ultraviolet Spectroscopy of Astrophysical Sources
}

\author{
99-LW-053 \\ Final Report \\ Christopher W. Mauche, Duane A. Liedahl, and Peter Beiersdorfer
}

\begin{abstract}
:
Work funded by the subject LDRD proposal has produced the following results. First, a comprehensive catalog of EUV lines from M-shell iron (Fe IX-XVI) in the 60-140 waveband. Second, a revised estimate of the radiative cooling of high-temperature plasmas by $\mathrm{Fe}$, which dominates the cooling in cosmic-abundance plasmas from $4 \times 10^{5} \mathrm{~K}$ to $1 \times 10^{7} \mathrm{~K}$. Third, laboratory data to correct theoretical atomic models and develop reliable spectral models of M-shell Fe in the EUV. Fourth, a solution of the origin of the quasi-continuum in EUV spectra of late-type stars, which has been variously ascribed to a high-temperature tail on the emission measure distribution of stellar coronae, reduced metal abundances, resonant scattering (destruction) of emission lines, and incompleteness of atomic models.
\end{abstract}

\section{Introduction}

As evidenced by the X-ray emission of our Sun, the cosmic X-ray background, and the more than 180,000 catalogued cosmic X-ray sources, the Universe is proficient at producing high-temperature plasmas. Diagnostics of the physical conditions of these cosmic plasmas is provided by spectra in the extreme ultraviolet (EUV; 912-100 $\AA_{\text {) and }}$ $\mathrm{X}$-ray $(0.1-10 \mathrm{keV})$ wavebands obtained with such facilities as the Extreme Ultraviolet Explorer (EUVE) and the Chandra X-ray Observatory $(C X O)$. The interpretation of these spectra requires large sets of atomic data and sophisticated spectral modeling capabilities, but all too often the atomic data required to develop these capabilities does not exist. To help remedy this situation, we conducted a project which combines experimental data from the Livermore Electron Beam Ion Trap (EBIT) facility with theoretical data generated with Livermore atomic physics codes to develop reliable spectral models of highly-charged ions in the EUV.

Due to its high cosmic abundance and persistence over a wide range of temperatures, iron $(\mathrm{Fe})$ is an important diagnostic of the plasma conditions in cosmic sources. For example, fully one third of the lines in the Solar EUV Rocket Telescope and Spectrograph catalog (Thomas \& Neupert 1994) of an average solar active region are due to Fe M-shell ions (Fe IX-XVII) which provide coverage of the temperature range from $6 \times 10^{5} \mathrm{~K}$ to $4 \times 10^{6} \mathrm{~K}$. The HULLAC (Hebrew University/Lawrence Livermore Atomic Code) atomic physics package (Klapisch et al. 1977; Bar-Shalom et al. 1988), can and has been used to calculate models of these ions, but these models (indeed, all such ab initio models) are 
compromised by electron-electron interactions. These do not seriously affect calculations of radiative and collisional rates, but they do affect wavelengths at the few percent level. For Fe IX for example, we find errors of up to $8 \AA$ for the $171.1 \AA 3 p^{6}-3 p^{5} 3 \mathrm{~d}$ resonance line, one of the strongest lines in the EUV spectrum of the Sun. Because of the high line density and line blending, these errors seriously compromise our ability to interpret the EUV spectra of cosmic sources. To remedy this situation, we performed a series of controlled laboratory measurements of the line emission from Fe IX-Fe XVII in the 60-140 Å waveband.

\section{EBIT Measurements}

The measurements were carried out with the Livermore electron beam ion trap (EBIT) facility using a spectrometer consisting of a variable line space grating and a thinned, back-illuminated, $\mathrm{LN}_{2}$-cooled CCD camera. The resolution of the spectrometer is $0.3 \AA$ at $100 \AA$, comparable to that of $E U V E$, and the electron density is $5 \times 10^{11} \mathrm{~cm}^{-3}$, comparable to that of the corona of the Sun and other late-type stars. By setting the electron beam to a particular energy, it is possible to select a specific charge state for spectroscopic study. For example, because the ionization potentfals of Fe VII, Fe VIII, ahd Fe IX are respectively 125,151 , and $233 \mathrm{eV}$, we are able to measure the emission spectra of $\mathrm{Fe}$ VIII, Fe IX, and Fe X by setting the electron beam energy to respectively 140, 200, and $250 \mathrm{eV}$. The resulting spectra are shown in Figures 1-3 with line identifications from the MEKAL database. In addition to the numerous strong emission lines missing from the database, the spectra reveal the presence of a quasi-continuum of emission due to unresolved weak emission lines of Fe IX (Fig. 2) and Fe X (Fig. 3). Figure 4 emphasizes the inability of the MEKAL database to reproduce the observed spectra: the model includes all of the Fe VIII, Fe IX, and Fe X lines included in the MEKAL database with ion abundance ratios adjusted to match the intensity of the strongest lines in the experimental spectrum shown in Figure 3. The model misses several of the prominent lines and completely fails to reproduce the quasi-continuum formed by the mass of weak lines. In fact, the MEKAL database accounts for less than $40 \%$ of the flux in Figure 1 and less than $31 \%$ of the flux in Figures 2 and 3 . These results feed directly into a revised estimate of the radiative cooling of high-temperature plasmas by $\mathrm{Fe}$, which dominates the cooling in cosmic-abundance plasmas from $4 \times 10^{5} \mathrm{~K}$ to $1 \times 10^{7} \mathrm{~K}$. 


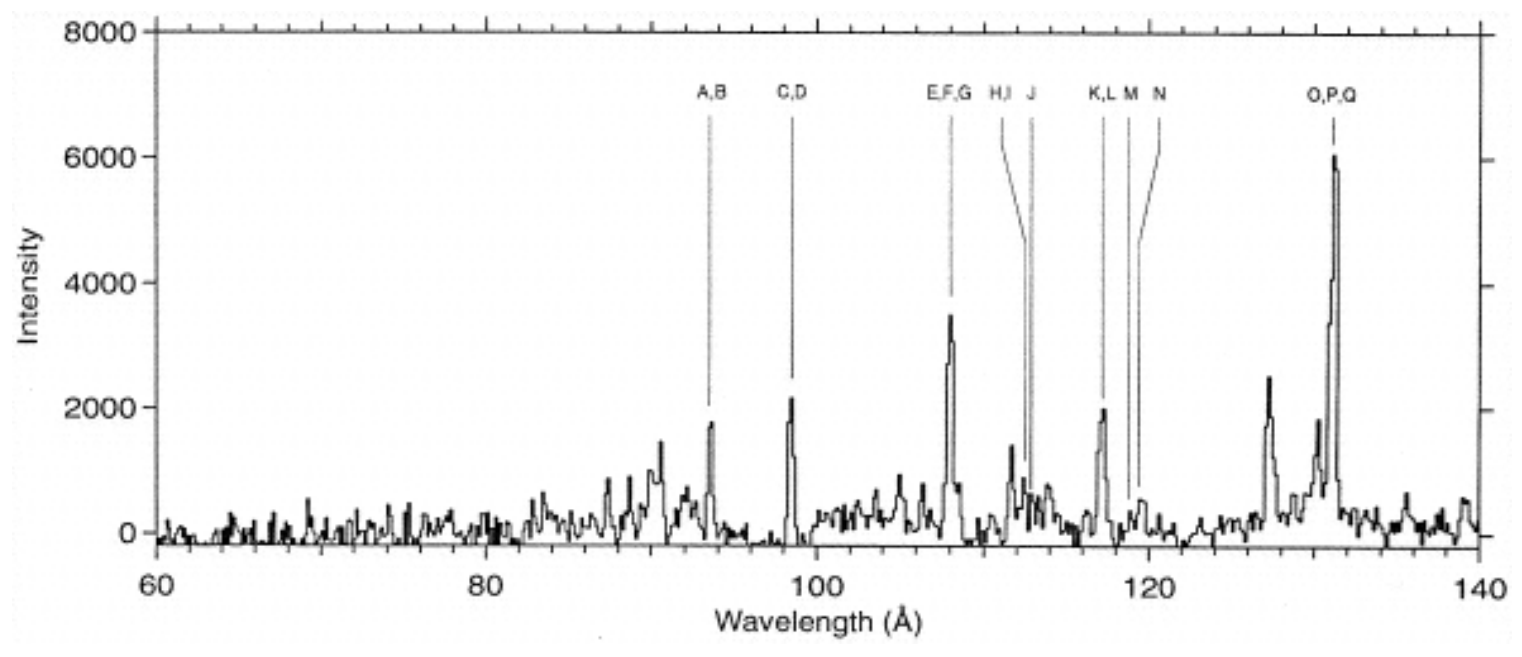

Fig. 1 - Line emission spectrum of Fe VII and Fe VIII ions excited at a beam energy of $140 \mathrm{eV}$. Fe VIII features that can be correlated with the MEKAL database are labeled with uppercase letters. Unlabeled features are not in the database.

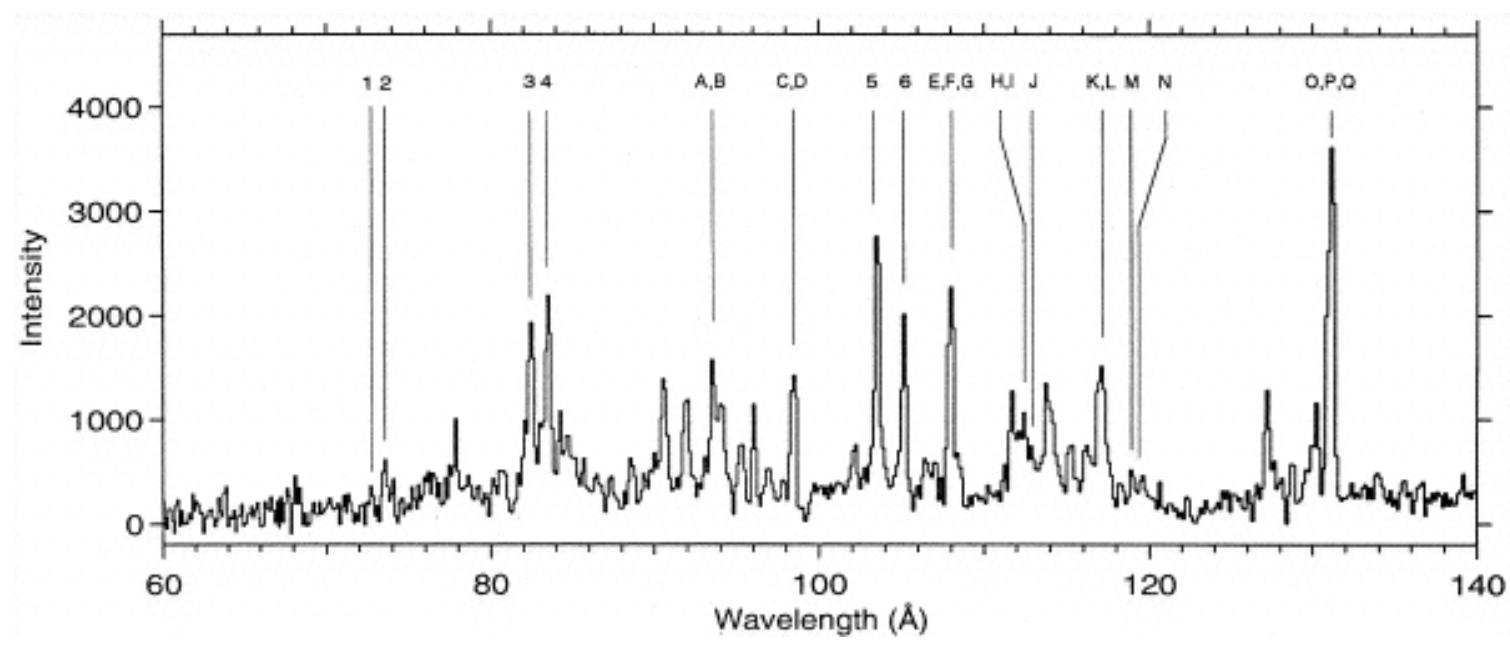

Fig. 2 - Line emission spectrum of Fe VII, Fe VIII, and Fe IX ions excited at a beam energy of $200 \mathrm{eV}$. Features that can be correlated with the MEKAL database are labeled with uppercase letters (Fe VIII) and Arabic numerals (Fe IX). Unlabeled features are not in the database. 


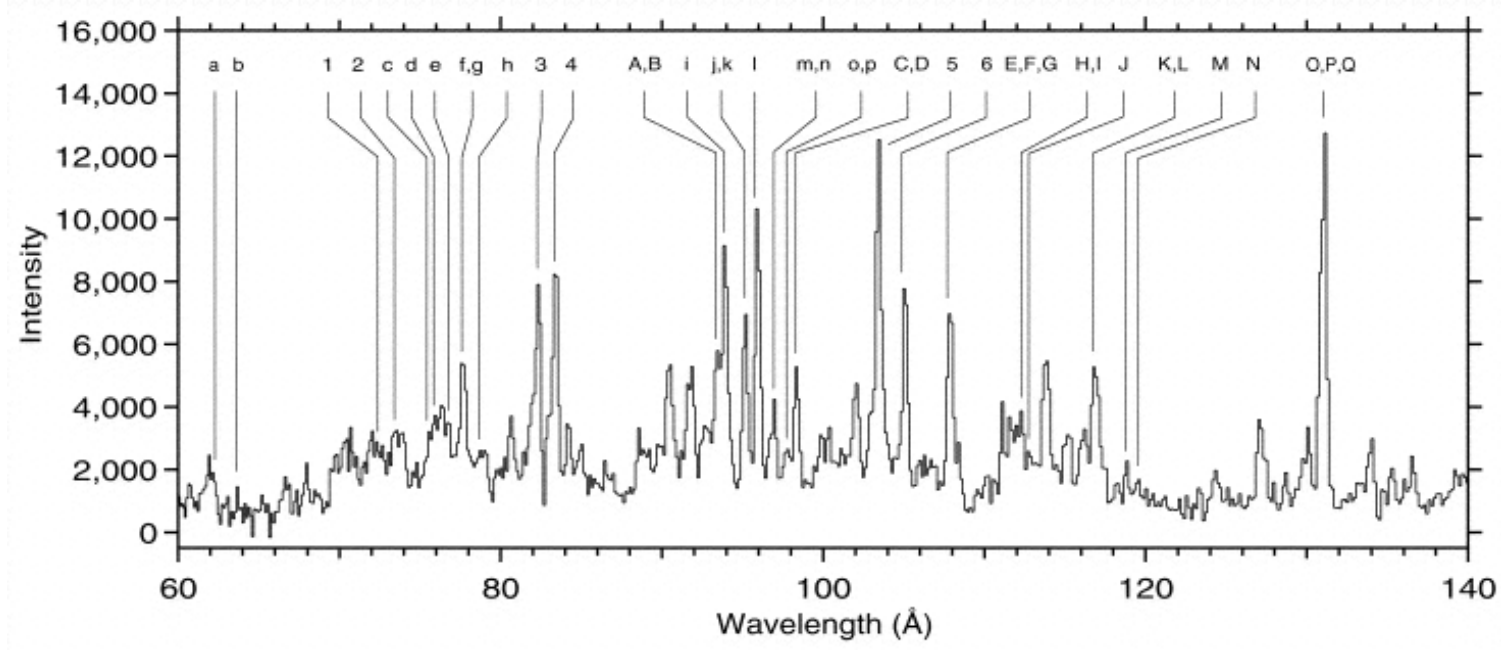

Fig. 3 - Line emission spectrum of Fe VII, Fe VIII, Fe IX, and Fe X ions excited at a beam energy of $250 \mathrm{eV}$. Features that can be correlated with the MEKAL database are labeled with uppercase letters (Fe VIII), Arabic numerals (Fe IX), and lowercase letters (Fe IX). Unlabeled features are not in the database.

We have used these EBIT measurements to produce a comprehensive catalog of EUV lines from M-shell Fe (Fe IX-XVI) in the 60-140 $\AA$ waveband and are using these data to correct theoretical atomic models and develop reliable spectral models of $\mathrm{M}$-shell $\mathrm{Fe}$ in the EUV.

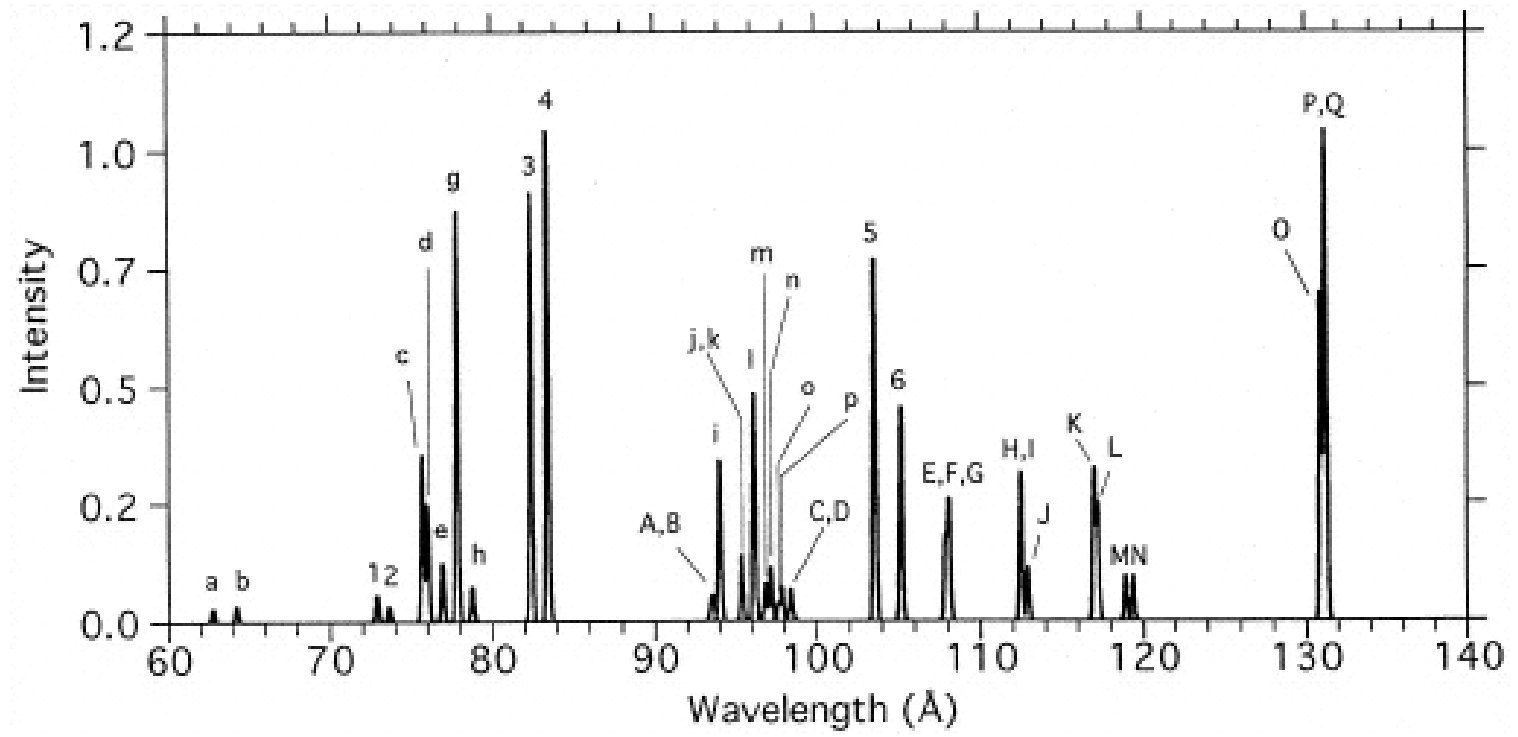

Fig. 4 - Simulated line emission spectrum using the MEKAL database. Features are labeled in the same notation used in Figs. 1-3. The relative ion abundances have been adjusted to simulate the experimental spectrum in Fig. 3. 


\section{Solution of the Origin of the EUV Quasi-Continuum of Late-Type Stars}

One of the outstanding problems in the study of the EUV spectra of late-type stars is the origin of the quasi-continuum or, equivalently, the low line-to-continuum ratio. A solution proposed by Mewe et al. (1995) is the presence of a high-temperature tail on the differential emission measure distribution with a temperature $T>10^{7.5} \mathrm{~K}$ which provides a strong bremsstrahlung continuum in the EUV waveband (Fig 5). However, such a hightemperature tail is inconsistent with higher-energy ROSAT spectra (Schmitt et al. 1996). Another possibility is that the metal abundances of most stellar coronae are below (solar) photospheric values. While this solution cannot be ruled out, it would imply a coronal abundance effect which is opposite to that seen in the Sun, in which elements like Fe with first ionization potentials below $10 \mathrm{eV}$ are enhanced relative to the photosphere. Another, yet more controversial resolution of the problem was offered by Schrijver et al. (1994), who suggested that resonant scattering of emission lines may be the cause of the low lineto-continuum ratio. This scenario has been disputed by numerous authors, who argue that a significant number of weak and closely spaced lines, not yet included in plasma codes, could form a quasi-continuum at short EUV wavelengths. Our EBIT measurements provide a direct, if (at this stage) qualitative, proof that the numerous weak emission lines of Fe form a quasi-continuum which can explain the low line-to-continuum ratio the EUV spectra of late-type stars.

\section{Acknowledgement:}

This work was performed under the auspices of the U.S. Department of Energy by University of California Lawrence Livermore National Laboratory under contract No. W-7405-Eng-48.
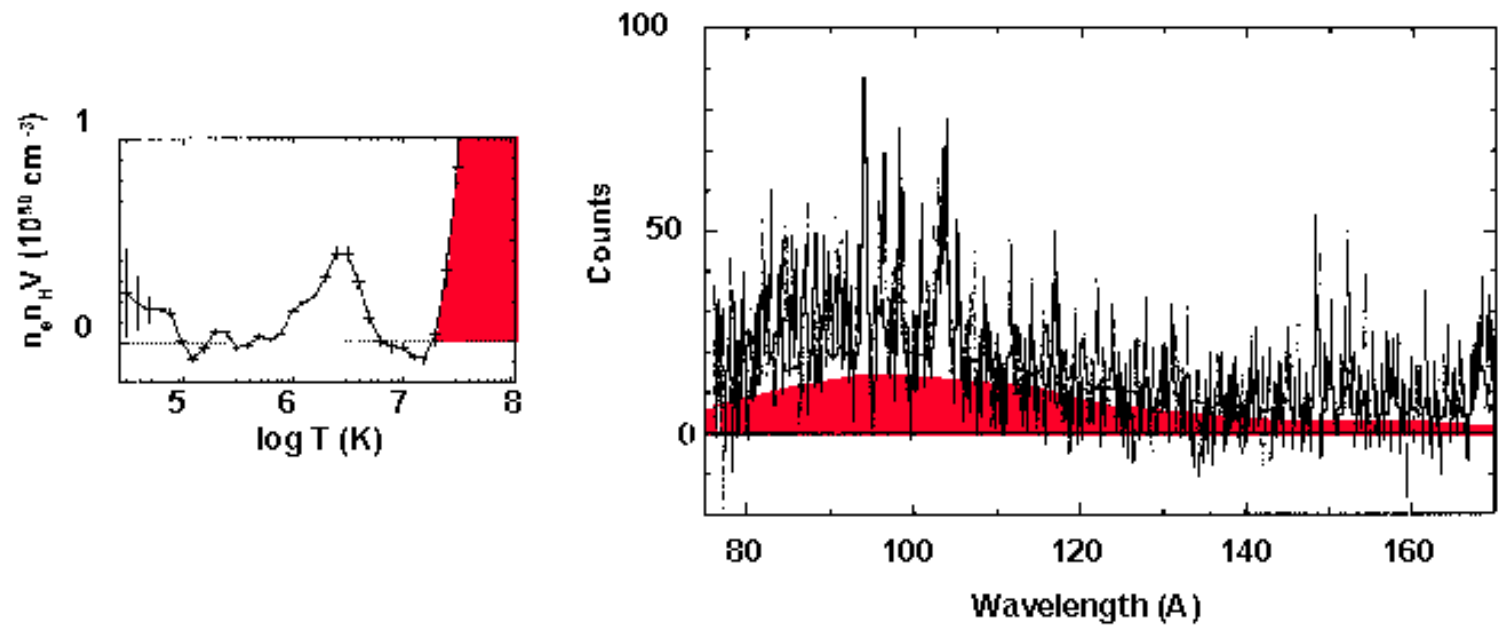

Fig. 5 - EUVE spectrum of the late-type star $\alpha$ Cen, the inferred differential emission measure distribution (DEM), and the contribution to the spectrum of the high-temperature tail of the DEM required by the strong quasi-continuum (shaded).

\section{References:}

Bar-Shalom A., Klapisch, M., \& Oreg J. 1988, Phys. Rev. A, 38, 1773. 
Klapisch, M.., Schwab, J. L., Fraenkel, J. S., \& Oreg, J. 1977, J. Opt. Soc. Am., 61, 148. Mewe, R., et al. 1995, A. \& Ap. Suppl., 296, 477.

Schmitt, J. H. M. M., Drake, J. J., \& Stern, R. A. 1996, Ap. J. (Letters), 465, L51.

Schrijver, C. J., van dev Oord, G. H. J., \& Mewe, R. 1994, A. \& Ap., 289, L23.

Thomas, R. J., \& Neupert, W. M. 1994, Ap. J. Suppl., 91, 461.

\section{Publications supported by this LDRD Project:}

"Cataloguing Emission Line Spectra from Fe VII-Fe XXIV in the Extreme Ultraviolet," Lepson, J. K., Beiersdorfer, P., Brown, G. V., Kahn, S. M., Liedahl, D. A., Mauche, C. W., \& Utter, S. B. 2000, Revista Mexicana de Astronomia y Astrofisica, in press [UCRLJC-136950].

"Observation of Quasi-Continuum Line Emission from Fe VII-X in the Extreme Ultraviolet Region below 140 Å,” Beiersdorfer, P., Lepson, J. K., Brown, G. V., Utter, S. B., Kahn, S. M., Liedahl, D. A., \& Mauche, C. W. 1999, Astrophys. Journal, 519, L185 [UCRL-JC-133895].

"Spectral Catalogue of the Intermediate Ionization Stages of Iron in the Extreme Ultraviolet," Beiersdorfer, P., Utter, S. B., Brown, G. V., Liedahl, D. A., Mauche, C. W., Kahn, S. M., Brickhouse, N. S., Dupree, A. K., Mewe, R., \& Kaastra, J. S. 1999, in Proceedings of the Laboratory Space Science Workshop, 1998 April 1-3, HarvardSmithsonian Center for Astrophysics, p. 89

[UCRL-JC-130832].

"Cataloguing the Lines from the Intermediate Charge States of Iron, Fe VII through Fe XXIV, in the Extreme Ultraviolet below $150 \AA$ A," Lepson, J. K., Beiersdorfer, P., Brown, G. V., Liedahl, D. A., Mauche, C. W., Utter, S. B., \& Kahn, S. M. 1999, Bull. Amer. Astro. Soc., 31, \#2, 706 [UCRL-JC-133800-ABS].

"Quasi-Continuum Line Emission from Fe VII-X in the Extreme Ultraviolet Region below 120 Å,” Beiersdorfer, P., Lepson, J. K., Brown, G. V., Liedahl, D. A., Mauche, C. W., Utter, S. B., \& Kahn, S. M. 1999, Bull. Amer. Astro. Soc., 31, \#2, 706

[UCRL-JC-133902-ABS]. 